improvements over placebo were seen in $0.03 \mathrm{mg}$ and $0.07 \mathrm{mg}$ treatment arms, achieving statistical significance for PTGA and MDGA. Further studies to identify relevant sub-populations and evaluate the safety and efficacy of SM04690 are ongoing.

Disclosure of Interest: Y. Yazici Shareholder of: Samumed, LLC, Employee of: Samumed, LLC, A. Gibofsky Shareholder of: AbbVie, Amgen, J\&J, GSK, Regeneron, Consultant for: AbbVie, Pfizer, Horizon, Iroko, Celgene, Novartis/Sandoz, Samumed, Speakers bureau: AbbVie, Amgen, Celgene, Pfizer, N. Lane Consultant for: Samumed, LLC, N. Skrepnik Grant/research support from: Samumed, LLC, Consultant for: Orthofix and Sanofi, E. Armas Grant/research support from: Samumed, LLC, C. Swearingen Shareholder of: Samumed, LLC, Employee of: Samumed, LLC, A. DiFrancesco Shareholder of: Samumed, LLC, Employee of: Samumed, LLC, J. Tambiah Shareholder of: Samumed, LLC, Employee of: Samumed, LLC, T. McAlindon Grant/research support from: Samumed, Consultant for: Astellas, Flexion, Pfizer, Regeneron, Samumed,and Seikugaku

DOI: 10.1136/annrheumdis-2017-eular.6382

\section{SAT0553 DETECTION OF SERUM LEVEL CHANGES OF MATRIX METALLOPROTIENASE-13 AND INTER LEUKIN-1 BETA DURING REMISSION AND FLARE-UPS OF PRIMARY OSTEOARTHRITIS OF THE KNEES}

Y. Hussein Gazar, H. Bassiouni. Alazhar University Hospital, Cairo, Egypt

Background: The diagnosis of osteoarthritis is currently based on radiographic criteria (eg, joint space width) and clinical symptoms (eg, pain and loss of function).

The evaluation of new disease-modifying osteoarthritis drugs (DMOADs) is performed on the same basis, since the regulatory bodies currently require evidence for an impact on radiographic joint space narrowing (JSN) and an impact on symptoms However, the limitations of radiography have led to research into alternative parameters for monitoring osteoarthritis that could serve as biomarkers in drug development.

Objectives: Detection the serum level of MMP-13 and IL-1 $\beta$ In OA of the knee during remission and exacerbation and if these Biomarkers can be validated as gold biomarkers in assessing OA progression and drug development in $\mathrm{OA}$ treatment.

Methods: This study was performed on 60 patients with knee osteoarthritis, 18 males $(30 \%)$ and 42 females $(70 \%)$, all diagnosed as osteoarthritis of one or both knees. Their ages ranged from (40 -65) years. The duration of their disease ranged from one to $15 y$ years. The control groups were 8 males (32\%) and 17 females $(68 \%)$. their ages ranged from (40-65) years.

- The patients were allowed to continue on the medications that they have pro inflammatory cytokines (IL-1 $\beta$ ) and degradative enzymes (MMP-13) are measured.

- Clinical assessing for pain using visual analogue scale (0-10)

- Assessing for pain, stiffness and physical functions by:

(A) the WOMAC osteoarthritis index

(B) Lequesne's algo functional index

- Asssesing the flare-ups using Knee Osteoarthritis Flare Ups Score (KOFUS).

Results: Patients who had 3 flare-ups (during one year follow up) showed the statistically significantly highest mean IL-1 \& \& MMP13 level.

There was no statistically significant difference between patients with no flare-up, 1 flare-up and 2 flare-ups; all showed statistically significantly lower mean levels.

There was a statistically significant positive (direct) correlation between IL-1 $\beta$, disease duration, KL, VAS, stiffness score, pain score, functional score, WOMAC and KOFUS. An increase in all these variables is associated with an increase in IL-1 $\beta$ \& MMP13.

Conclusions:

- There is a potential role for IL1 beta and MMP 13 biomarkers in assessing the development in osteoarthritis.

- IL $1 \beta$ and MMP 13 were founded to be correlated positively in patients with knee OA this correlation sounded right as the expression of MMP 13 depends on the level of IL1 $\beta$.

- Although all medications groups failed to lower the level of IL $1 \beta$ and MMP 13 ,yet there was a numerical difference in favor of Diacerine and NSAID. - patients on both Diacerine and NSAID had the lowerest rate of flare ups

- It is recommended that the early measurement of biomarkers may detect cases to progress and thus stronger treatment may be given for these groups.

Disclosure of Interest: None declared

DOI: 10.1136/annrheumdis-2017-eular.1134

\section{SAT0554 INVESTIGATION OF SELECTED BIOCHEMICAL MARKERS IN KNEE OSTEOARTHRITIS: THE FRAMINGHAM OSTEOARTHRITIS COHORT}

Y. Luo $^{1}$, Y. He ${ }^{1}$, A.S. Siebuhr ${ }^{1}$, S. Hoielt ${ }^{1}$, D. Felson ${ }^{2}$, M. Karsdal ${ }^{1}$, A.-C. Bay-Jensen ${ }^{1} .{ }^{1}$ Biomarkers \& Research, Nordic Bioscience, Herlev, Denmark; ${ }^{2}$ Clinical Epidemiology Research and Training Unit, Boston University School of Medicine, Boston, United States

Background: Osteoarthritis $(\mathrm{OA})$ is a major cause of functional impairment and disability among the elderly. There is an unmet need for the development of biomarkers for identifying patients with high risk for $\mathrm{OA}$ and for monitoring drug efficacy. Specific and sensitive biochemical markers revealing the turnover of bone, cartilage, and synovial tissue may be useful for investigation and monitoring of $\mathrm{OA}$.

Objectives: To investigate a targeted set of five biochemical markers, which reflect joint tissue turnover, for their ability to evaluate the prevalence of radiographic and symptomatic knee osteoarthritis $(\mathrm{OA})$ in a substudy from the cross-sectional Framingham OA cohort (FOA).

Methods: The subjects from the community-based FOA cohort were divided up based on a terminology proposed by the FNIH-OAI consortium. Two main groups were defined: subjects with radiographic knee OA (RKOA, n=80) and a group with no radiographic OA (NRKOA, $n=136$ ). The presence of ROA as any Kellgren-Lawrence $(\mathrm{KL})$ grade $=2$ or 3 . The RKOA group were further divided into two groups; those with persistent symptoms of a joint (RKOA+S, n=30) and those without (RKOA-S, $n=50)$. $+S$ was defined as having pain, aching or stiffness in either knee on most days.

Serum levels of C1M, CRPM and huARGS (matrix metalloproteinases cleaved type I collagen and C-reactive protein neo-epitopes, aggrecanase cleaved ${ }^{374}$ ARGS neoepitope of aggrecan, Nordic Bioscience) were determined by ELISA. Serum levels of cartilage synthesis and degradation biomarkers, hsPro$\mathrm{C} 2$ and hsAGNx-1 (procollagen type IIB N-terminal propeptide, aggrecanase cleaved TEGE ${ }^{373}$ neoepitope of aggrecan, Nordic Bioscience) were measured by electrochemiluminescence immunoassay (ECLIA). Each measure was fisher transformed in order to be comparable across the biomarkers. The correlation between the biomarkers and covariates was assessed.

The subjects of substudy were segregated into two groups based on the cut-off values of each biomarker. The cut-off values of these markers were set as mean of their reference levels. We used logistic regression to compare these two groups, and to examine the association between each marker and the presence of $O A$ and/or pain. All confounding factors were adjusted.

Results: The two main groups were well-matched by age, sex, and BMI. Two biomarkers correlated negatively with BMI: C1M and CRPM. Aggrecan degradation biomarker, hsAGNx-1 was negatively associated with age while the huARGS was not associated with it (Table 1).

CRPM was associated with a lower risk of RKOA+S. Interestingly, hsPro-C2 was associated with a higher risk of it (Table 2).

Table 1. Subject characteristics of substudy

\begin{tabular}{lcccc}
\hline Covariates & $\begin{array}{c}\text { NRKOA } \\
(\mathrm{n}=136)\end{array}$ & $\begin{array}{c}\text { RKOA-S } \\
(\mathrm{n}=50)\end{array}$ & $\begin{array}{c}\text { RKOA+S } \\
(\mathrm{n}=30)\end{array}$ & $\begin{array}{c}\text { Biomarker (Fisher transformed) } \\
\text { associations with covariates }\end{array}$ \\
\hline BMI, mean kg/m $\mathrm{m}^{2}(\mathrm{SD})$ & $31.3(3.79)$ & $32.3(6.05)$ & $31.6(5.12)$ & C1M, CRPM \\
WOMAC pain & $0.182(0.657)$ & $0.820(1.66)$ & $4.83(3.64)$ & \\
Age, mean years (SD) & $62.6(8.18)$ & $63.7(7.06)$ & $62.4(7.09)$ & hsAGNx-1 \\
Sex, $\mathrm{n}(\%)$ female & $82(60.3 \%)$ & $33(66.0 \%)$ & $18(60.0 \%)$ & C1M \\
\hline
\end{tabular}

\begin{tabular}{|c|c|c|}
\hline Biomarker & OR of RKOA-S $(95 \% \mathrm{Cl})$ & OR of RKOA+S $(95 \% \mathrm{Cl})$ \\
\hline C1M & $1.07(0.54 \cdot 2.10)$ & $0.47(0.18-1.24)$ \\
\hline CRPM & $0.97(0.51-1.88)$ & $0.41(0.16-1.02)$ \\
\hline hSAGNx-1 & $1.20(0.61 \cdot 2.38)$ & $0.68(0.27-1.72)$ \\
\hline hsPro-C2 & $1.27(0.63 \cdot 2.57)$ & $2.51(1.10-5.73)$ \\
\hline hUARGS & $1.43(0.71-2.88)$ & $1.19(0.50-2.84)$ \\
\hline Biomarker & OR of RKOA-S $(95 \% \mathrm{Cl})$ & OR of RKOA+S (95\% CI) \\
\hline C1M & $0.96(0.47-1.95)$ & $0.45(0.17-1.20)$ \\
\hline CRPM & $0.87(0.44 \cdot 1.71)$ & $0.39(0.16-1.00)$ \\
\hline hSAGNx-1 & $1.11(0.55-2.23)$ & $0.68(0.27-1.72)$ \\
\hline hsPro-C2 & $1.32(0.64 \cdot 2.72)$ & $2.55(1.11 \cdot 5.82)$ \\
\hline hUARGS & $1.49(0.73-3.03)$ & $1.21(0.50-2.92)$ \\
\hline
\end{tabular}

Conclusions: This study provides two major findings: 1) aggrecan degradation is not just aggrecan degradation and different neo-epitopes have distinct clinical relevance; 2) CRPM is a candidate biomarker of disease activity and for patient profiling. These data suggest a reference for interpretation of OA subject biomarker data in future human studies.

Disclosure of Interest: None declared

DOI: 10.1136/annrheumdis-2017-eular.1744

\section{SAT0555 MRI-DETECTED KNEE OSTEOPHYTE: NATURAL HISTORY AND STRUCTURAL RISK FACTORS AFFECTING CHANGE}

Z. Zhu ${ }^{1}$, W. Han $^{2}$, S. Zheng ${ }^{2}$, T. Winzenberg ${ }^{2}$, F. Cicuttini ${ }^{3}$, C. Ding ${ }^{2}$, G. Jones ${ }^{2}$ on behalf of Musculoskeletal Unit, Menzies Institute for Medical Research. ${ }^{1} 12779$, Hobart, Australia; ${ }^{2}$ Musculoskeletal Unit, 12779, Hobart; ${ }^{3}$ Department of epidemiology and Preventive Medicine, Monash University, Melbourne, Australia

Background: Although magnetic resonance imaging (MRI) has been proved to be far more sensitive than conventional radiographs to detect $O P$, the natural history of MRI-detected OP in older adults has not yet been described, and it is unclear whether knee structural abnormalities, including cartilage defects, cartilage volume, bone marrow lesions (BMLs), meniscal extrusion, infrapatellar fat pad (IPFP), and effusion-synovitis, can predict osteophyte change.

Objectives: To describe the natural history of knee MRI-detected OP, and to determine if knee structural risk factors are associated with change of MRI-detected OP in a longitudinal study of older adults. 
Methods: 413 randomly selected older adults (mean age 63 years) had magnetic resonance imaging at baseline and approximately 2.6 years later to measure knee OP, cartilage defect, cartilage volume, BMLs, meniscal extrusion, IPFP quality score/maximum area and effusion-synovitis. Weight, height, body mass index (BMI) and leg muscle strength were measured by standard protocols. Results: $85 \%$ participants had MRI-detected OP at baseline. Over 2.6 years, the average OP score increased significantly in all compartments. The OP score remained stable in $53 \%$ participants and worsened in $46 \%$ ( $\geq 1$-point increase) OP, with $1 \%$ decreasing. Baseline factors associated with an increase in MRI-detected OPs over 2.6 years included BMI, cartilage defects, BMLs, meniscal extrusion, IPFP quality score and Effusion. In multivariable analyses, baseline cartilage defects, BMLs and meniscal extrusions and IPFP quality score were site-specifically and significantly associated with increased OP at medial tibiofemoral, lateral tibiofemoral and total compartments ( $p$ all $<0.05$ ). In contrast, total and suprapatellar pouch effusion-synovitis were significantly associated with increased OP at total and lateral compartments ( $p$ all $<0.01$ ). The significant associations between baseline cartilage volume and increased OPs at medial and total compartments became non-significant after further adjustment for other knee structural abnormalities. Age sex and smoking status were not associated with increased OPs over time.

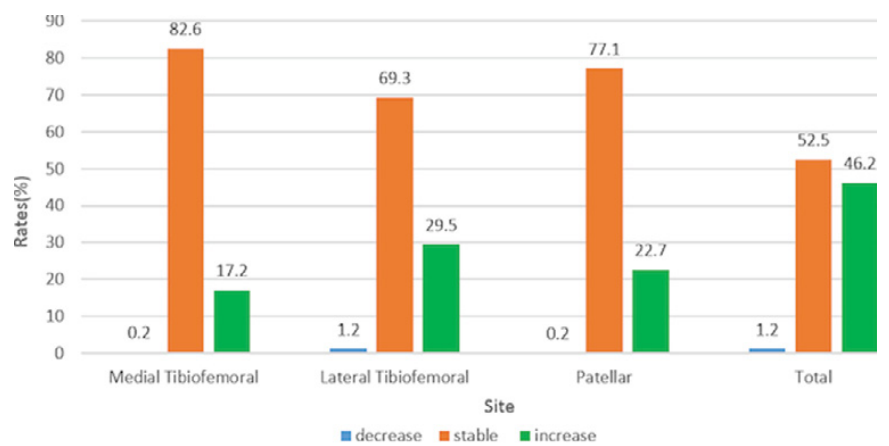

Figure 2. Change in MRI-detected osteophyte scores by site over 2.6 years. Total score was calculated by summing medial tibiofemoral, lateral tibiofemoral and patellar scores.

Conclusions: Knee MRI-detected OP in older adults is common and, in contrast to radiographs, is likely to progress over a relatively short period. Progression can be predicted by structural risk factors suggesting they are a consequence of these abnormalities.

References:

[1] McCauley TR, Kornaat PR, Jee WH. Central osteophytes in the knee: prevalence and association with cartilage defects on MR imaging. AJR Am J Roentgenol. $2001 \mathrm{Feb} ;$ 176(2):359-364.

Acknowledgements: The authors thank the participants who made this study possible, and acknowledge the role of the staff and volunteers in collecting the data, particularly research nurses Boon C and Boon P. Warren R assessed MRIs and $\mathrm{Dr}$ Srikanth $\mathrm{V}$ and $\mathrm{Dr}$ Cooley $\mathrm{H}$ assessed radiographs.

Disclosure of Interest: None declared

DOI: 10.1136/annrheumdis-2017-eular.1161

\section{SATURDAY, 17 JUNE 2017}

\section{Infection-related rheumatic diseases}

\section{SAT0556 RISK FACTORS FOR SEVERE INFECTION AND RATIONALE FOR IMMUNOGLOBULIN MONITORING DURING RITUXIMAB TREATMENT IN AUTOIMMUNE RHEUMATIC DISEASES}

M.Y. Md Yusof $^{1,2}$, E.M. Vital ${ }^{1,2}$, D. McElvenny ${ }^{3}$, E.M. Hensor ${ }^{1,2}$, S. Das ${ }^{1}$, M.H. Buch ${ }^{1,2}$, P. Emery ${ }^{1,2}$, S. Savic ${ }^{1,2}$. ${ }^{1}$ Rheumatology, Leeds Institute of Rheumatic and Musculoskeletal Medicine, University of Leeds; ${ }^{2}$ NIHR Leeds Musculoskeletal Biomedical Research Unit, Leeds Teaching Hospitals NHS Trust, Leeds; ${ }^{3}$ Institute of Population Health, University of Manchester, Manchester, United Kingdom

Background: Rituximab (RTX) has been used in the treatment of various autoimmune rheumatic diseases (AIRDs) for over a decade. Repeat cycles are effective for maintenance but may lead to hypogammaglobulinaemia. Low IgG at baseline has been associated with post-treatment infection rate but may be confounded by other clinical variables and fully adjusted models with method for handling missing data have not been presented. Importance of post-treatment change in lgs has also not been proven.

Objectives: To evaluate risk factors for severe infection in multivariable analysis and assess outcome of hypogammaglobulinaemia.

Methods: The first 700 consecutive patients with AIRDs treated with RTX at a single centre were studied. Each cycle of RTX consisted of $2 \times 1000 \mathrm{mg}$ infusions repeated on clinical relapse. $\lg \mathrm{M}, \lg A$ and $\lg G$ levels were measured at baseline and 4-6 months after each cycle. Multiple imputation was used for missing data. Baseline factors for predicting serious infection and low Ig were tested using univariable and multivariable (MVA) logistic regression analyses.
Results: 550 patients were female, median age (IQR) at RTX initiation 58 (46-68) years and median disease duration (IQR) $7.9(3.4-15.0)$ years. $506(72 \%)$ had RA, 94 (13\%) SLE, 49 (7\%) AAV, 14 (2\%) DM, 5 (1\%) APS, $6(1 \%)$ SSc and $26(4 \%)$ other CTD. 364 (52\%) were biologic-naïve and 515 (74\%) were on concomitant DMARDs. Total follow-up: 2940 patient-years (PY). 284 serious infections were recorded in 179 patients (9.7/100 PY); 88 cases within 12 months of cycle 1 (C1). In MVA, previous severe infection (OR 10.7, 95\% CI 5.8-19.5), low IgG (OR 3.6, 95\% Cl 1.5-8.6), previous cancer (OR 2.9, 95\% Cl 1.2-6.6) and chronic lung disease (OR 1.7, 95\% $\mathrm{Cl} 0.9-3.1$ ) increased the odds of a severe infection within 12 months of $\mathrm{C} 1$. A diagnosis of CTD was associated with lower risk (OR $0.5,95 \% \mathrm{Cl} 0.2-0.9$ ). Low $\lg$ at RTX initiation was predicted by older age, previous cancer, RA diagnosis, previous severe infection and previous treatment with cyclophosphamide. In C1-C3, higher rate of change in IgA and IgG levels were associated with serious infections (Figure 1). Overall, only $7(1 \%)$ of the patients required Ig replacement in this cohort

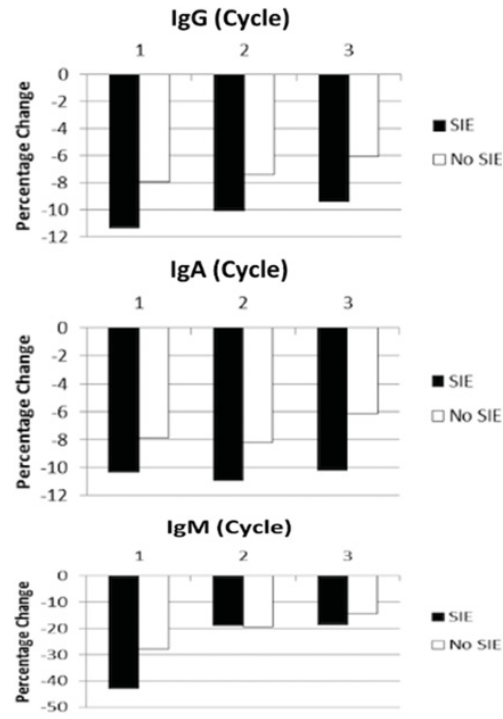

Conclusions: Factors associated with serious infection at RTX initiation include previous serious infection, low IgG, previous cancer, a diagnosis of RA and chronic lung disease. This is the first study to show the rationale for monitoring the rate of change in lg levels during repeat cycles of RTX, with reduction in all Ig subclasses being associated with increased risk of post-treatment infection. Further analysis including predictors of serious infections in repeat cycles is in progress and will be used to develop guidelines for safety monitoring of rituximab. Acknowledgements: This research was funded/supported by the National Institute for Health Research (NIHR) and NIHR Leeds Musculoskeletal Biomedical Research Unit based at Leeds Teaching Hospitals NHS Trust; (DRF-2014-07155). The views expressed are those of the author(s) and not necessarily those of the NHS, the NIHR or the Department of Health.

Disclosure of Interest: None declared

DOI: 10.1136/annrheumdis-2017-eular.6896

\section{SAT0557 CHIKUNGUNYA OUTBREAK IN BRAZIL: DEMOGRAPHIC AND CLINICAL CHARACTERIZATION OF 732 PATIENTS -} CHIKBRASIL COHORT

A.L. Duarte ${ }^{1}$, C.D.L. Marques ${ }^{1}$, P.R. Santos ${ }^{1}$, A. Ranzolin ${ }^{2}$, N.G. Cavalcanti ${ }^{1}$ R.S. Gonçalves ${ }^{3}$, A.T. Dantas ${ }^{1}$, M.S. Luna ${ }^{1}$, C.A. Andrade ${ }^{1}$, L.F. Rocha Junior ${ }^{3}$, M.R. Freitas ${ }^{3}$, P.R. Melo ${ }^{1}$, L.D. Valadares ${ }^{4}$, C.A. da Fonte ${ }^{4}$, M.L.D. Valadares ${ }^{4}$, E. Freire ${ }^{5}$, A.K.G. Melo ${ }^{5}$, M.M. Medeiros ${ }^{6}$, M.C. Bezerra ${ }^{6}$, V.B. Marques ${ }^{6}$, R.A. Océa ${ }^{7}{ }^{1}$ Internal Medicine, Universidade Federal de Pernambuco:

${ }^{2}$ Rheumatology; ${ }^{3}$ Internal Medicine, Instituto de Medicina Integral Prof. Fernando Figueira; ${ }^{4}$ Rheumatology, Hospital Getulio Vargas, Recife; ${ }^{5}$ Rheumatology, Universidade Federal da Paraíba, João Pessoa; ${ }^{6}$ Rheumatology, Universidade Federal do Ceará, Fortaleza; ${ }^{7}$ Rheumatology, Universidade Federal de Sergipe, Aracaju, Brazil

Background: Chikungunya Fever (CF) is a disease characterized by acute febrile arthritis and caused by a mosquito-transmitted alphavirus. Considering the wide distribution of the vector, the presence of imported cases from 2010 and Brazilian population's susceptibility, there was a dispersal and establishment of Chikungunya virus (CHIKV) throughout the country. Since 2014, the CF has achieved a large proportion of the Brazilian population and has been responsible for the development of chronic joint symptoms in thousands of people.

Objectives: To describe the demographic, clinical and serological characteristics of patients from specialized Rheumatology services from northeastern Brazil, in a large, multicenter cohort.

Methods: Data from 732 patients in a prospective, multicenter, observational cohort conducted in six research rheumatology centers were analyzed. Patients 